\title{
Phase II trial of capecitabine combined with docetaxel in previously treated patients with non-small cell lung cancer: A randomized controlled study
}

\author{
QIANG LIN $^{1 *}$, FAN-JIE MENG ${ }^{1}$, YUE'E LIU $^{1}$, NA WANG $^{1}$, XIAO-CANG REN $^{1}$, XUE-JI CHEN ${ }^{1}$, \\ XIAO-HUI GE ${ }^{2}$, CHAO-XING LIU ${ }^{3}$, JING HU ${ }^{1}$, DONG-YING WANG ${ }^{1}$, BIN CAO ${ }^{1}$ and YANNAN ZHAO ${ }^{1}$ \\ ${ }^{1}$ Department of Oncology, Hebei Medical University Affiliated North China Petroleum Bureau General Hospital, \\ Renqiu 062552; ${ }^{2}$ Department of Radiation Oncology, Hebei University Affiliated Hospital, Baoding, Hebei 071000; \\ ${ }^{3}$ Department of Radiation Oncology, Beijing University First Hospital, Beijing 10034, P.R. China
}

Received November 14, 2011; Accepted January 12, 2012

DOI: $10.3892 / \mathrm{ol} .2012 .575$

\begin{abstract}
Docetaxel alone has been confirmed to be beneficial to patients with advanced previously treated non-small cell lung cancer (NSCLC). However, the duration and survival time is short. The study of two-agent combination regimens has important clinical significance. We conducted this randomized controlled phase II trial to comparatively evaluate the efficacy and side effects of capecitabine combined with docetaxel in previously treated patients with NSCLC. Patients with previously treated NSCLC who failed first-line chemotherapy were randomized into two groups; one received capecitabine combined with docetaxel (XT group) and the other received docetaxel alone (T group). Patients in the XT group received chemotherapy as follows: capecitabine $625 \mathrm{mg} / \mathrm{m}^{2}$, p.o. bid, days 5-18; and docetaxel $30 \mathrm{mg} / \mathrm{m}^{2}$, days 1 and 8 , while patients in the T group received docetaxel $35 \mathrm{mg} / \mathrm{m}^{2}$ on days 1 and 8 . The primary endpoint was time to progression (TTP), and secondary endpoints were overall survival (OS), response rate (RR) and disease control rate (DCR). Forty-eight patients were recruited (23 in the XT group and 25 in the T group). TTP, median survival time (MST) and 1-year OS rate in the XT group and the T group were 7 months, 12 months, $47.6 \%$ and 3 months, 12 months, $39.6 \%$, respectively. The TTP in the XT group was significantly longer compared to that in the T group $\left(\chi^{2}=4.763, p=0.029\right)$. The RR and DCR in the XT group and T group were $13.0 \%(3 / 23), 78.3 \%(18 / 23)$ and $12.0 \%(3 / 25), 76 \%(19 / 25)$, respectively. The difference was not significant $(\mathrm{p}>0.05)$. The major side effects observed in the
\end{abstract}

Correspondence to: Dr Qiang Lin, Department of Oncology, Hebei Medical University Affiliated North China Petroleum Bureau General Hospital, 8 Huizhan Avenue, Renqiu City, Hebei 062552, P.R. China

E-mail: zyy_linqiang@hotmail.com

Key words: non-small cell lung cancer, chemotherapy, second-line therapy, capecitabine, docetaxel two groups were neutropenia, fatigue and nausea, and toxicities were mild to modest. No severe cases of hand-foot syndrome were observed in the XT group. In conclusion, compared with docetaxel alone, capecitabine combined with docetaxel for patients with previously treated NSCLC achieved a significantly longer TTP and this regimen was well tolerated. The relatively high median TTP, 1-year OS rate and DCR encourage further evaluation of this regimen in a randomized phase III trial.

\section{Introduction}

Global cancer statistics show that the morbidity and mortality rates of lung cancer in 2008 were the highest of all cancer types. In that year, 1,600,000 new cases occurred, and 1,400,000 patients succumbed (1). Non-small cell lung cancer (NSCLC) accounts for approximately $80 \%$ of all lung cancers, and most cases are at an advanced stage and unresectable when diagnosed clinically (2). The two-agent platinum-based regimen is the standard first-line chemotherapy regimen for advanced patients. However, most patients need to receive second-line chemotherapy due to relapse or metastasis following first-line chemotherapy. The guidelines of the National Comprehensive Cancer Network (NCCN) in the USA recommend that the standard second-line chemotherapy regimen of NSCLC includes docetaxel alone and pemetrexed alone (3). Of these, docetaxel is the first chemotherapy medicine proven to be better than best supportive treatment (BST) in second-line treatment. Therefore, its application is widespread and well-developed. However, treatment efficiency of docetaxel in the second-line treatment of NSCLC is unsatisfactory. Its overall response rate (RR) is $14-25 \%$, the median survival time (MST) is 6.1-9.5 months, and the 1-year overall survival rate is only $23-44 \%$ (4). Therefore, exploring the effect of a two-agent combination chemotherapy regimen in previously treated patients with NSCLC has important clinical significance. As the precursor medicine of fluorouracil, capecitabine has been proven to be effective for treating various types of cancer. In addition, capecitabine and docetaxel have a synergistic antitumor effect. In breast cancer, capecitabine combined with docetaxel demonstrated a longer time to progression (TTP) 
and better overall survival (OS) than docetaxel alone (5). Both theoretical and clinical research prove that a combination of the two is effective in the treatment of NSCLC (6-9). However, to the best of our knowledge, there is no study comparing the efficacy of capecitabine combined with docetaxel and docetaxel alone in previously treated patients with NSCLC. Therefore, we conducted a randomized controlled phase II trial to comparatively evaluate the efficacy and side effects of capecitabine combined with docetaxel in previously treated patients with NSCLC.

\section{Patients and materials}

Eligibility. Patients with histologically confirmed advanced NSCLC, who had received at least one platinum-based chemotherapy regimen as treatment for their NSCLC and had shown disease progression, were eligible to participate. Prior to enrollment in the study, a minimum of 21 days had to have elapsed since any prior chemotherapy. Eligibility criteria also included the following: age $\geq 18$ and $\leq 75$ years; Karnofsky performance status $\geq 60$; life expectancy $\geq 3$ months; at least one evaluable lesion; adequate hematopoietic function (absolute neutrophile count $\geq 2.0 \times 10^{9} / 1$, platelet count $\geq 100 \times 10^{9} / 1$ and hemoglobin count $\geq 100 \mathrm{~g} / \mathrm{l}$ ); adequate hepatic and renal function (serum creatinine, aspartate aminotransferase, alanine aminotransferase and total serum bilirubin $\leq$ upper limits of normal).

The exclusion criteria consisted of any of the following: pregnancy; lactation; a history of other malignancies, with the exception of carcinoma in situ of the cervix, non-melanomatous skin cancer, or cancer from which the patient had not been disease-free for five years; brain metastases that were not neurologically stable; a general medical condition preventing combined chemotherapy; as well as use of any other concurrent antineoplastic therapy.

Pretreatment evaluation. Pretreatment evaluation included medical history, complete physical examination, chest and abdominal helical CT scan, electrocardiography, bone marrow scan (if clinically indicated), complete blood count and biochemical profile. These pretreatment tests were performed during the week prior to treatment initiation. Patients received physical examinations, and blood counts were performed once a week or more often if necessary. A biochemical profile was obtained and electrocardiography performed prior to each chemotherapy cycle.

Ethics. The procedures were approved by the Ethics Committee of Hebei Medical University and were performed in accordance with the ethics standards of human experimentation and with the Helsinki Declaration of 1975, as revised in 2000. The patients provided written informed consent.

Chemotherapy. Previous studies of human cancer xenografts showed that docetaxel upregulated thymidine phospharylase (TP) activity, and that docetaxel combined with capecitabine had a synergistic effect (6). Certain phase II trials have verified the preliminary efficacy and safety of capecitabine combined with docetaxel $(7,8)$. As Chinese and Korean all belong to the Oriental population in Asia, our study adopted the recommended dose of the research on Koreans of a phase II trial (8).
The preclinical study indicated that the effect of docetaxel on the upregulation of TP activity was strongest between days 6 and 10 following administration, which was proven by clinical trials $(6,7)$. Therefore, we adjusted the administration time of the Korean regimen; subsequently, capecitabine was orally administered initially on day 5 of treatment instead of day 1 . Results of certain studies showed that weekly docetaxel administration not only obtained the same treatment efficiency, but also significantly reduced the risk of neutropenia (10). Therefore, in our study, the weekly administration regimen was adopted for docetaxel, i.e., docetaxel was administered on days 1 and 8 of treatment (11).

Patients were randomized into 2 treatment groups: the capecitabine combined with docetaxel group (XT group) or the docetaxel alone group ( $\mathrm{T}$ group). In the XT group, patients received two-agent chemotherapy: capecitabine $625 \mathrm{mg} / \mathrm{m}^{2}$, p.o. bid, on days 5-18, repeated every 3 weeks. The precise dose of capecitabine was calculated by body surface area and rounded off to the nearest $100 \mathrm{mg}$ that would allow equal morning and evening doses. Docetaxel $30 \mathrm{mg} / \mathrm{m}^{2}$ was administered as a 1-h intravenous infusion on days 1 and 8 of each 3 -week cycle along with capecitabine. In the $\mathrm{T}$ group, patients received single-agent chemotherapy: docetaxel $35 \mathrm{mg} / \mathrm{m}^{2}$ was administered as a 1-h intravenous infusion on days 1 and 8 of each 3 -week cycle. All patients in the two groups were premedicated with oral dexamethasone: $8 \mathrm{mg}$, p.o. bid, d-1, d1, d2 and d7, d8, d9. Up to 6 cycles of chemotherapy were administered to patients in the two groups.

To ensure the continuity of chemotherapy, we carried out supportive treatments by promotion of neutrophile and platelets, anemia correction, antiemetics and intravenous rehydration, for example, if indicated clinically.

Dose attenuation. Prior to starting a new cycle of treatment, an absolute neutrophil count of $\geq 2.0 \times 10^{9} / 1$, a platelet count $\geq 100 \times 10^{9} / 1$ and an improvement to grade I or lower in other treatment-related toxicities was required. Different criteria for dose adjustment within a cycle and dose modification for subsequent cycles of treatment were adopted.

Capecitabine was withheld for grade II-III hand-foot syndrome (HFS) until regression to grade I or lower and then restarted at the same dose for grade II HFS or at $75 \%$ of the initial dose level for grade III HFS. For other toxicities, capecitabine was withheld within a cycle for grade III or higher non-hematological (except grade III nausea and isolated hyperbilirubinemia) or grade III or higher hematological toxicity. Capecitabine was resumed at the original dose level for grade II non-hematological or grade III hematological toxicity, or with $25 \%$ dose reduction for grade III or higher non-hematological (except grade III nausea and isolated hyperbilirubinemia) or grade IV hematological toxicity. Capecitabine was not interrupted for isolated hyperbilirubinemia.

Docetaxel on day 8 was withheld for grade II or higher hyperbilirubinemia or any other grade III or higher toxicity (except grade III nausea) during the scheduled day of docetaxel administration and the patient was re-evaluated weekly until symptoms regressed to grade II or lower. Missed doses of docetaxel were not made up. The following cycle of treatment was restarted at the same dose for grade III toxicity and was reduced by $25 \%$ for grade IV toxicity during the subsequent cycle. 
Table I. Patient characteristics.

\begin{tabular}{lcc}
\hline Characteristics & XT group & T group \\
\hline Gender & 14 & \\
Male & 9 & 15 \\
Female & & 10 \\
Age (years) & $48-73$ & $50-71$ \\
Range & 65 & 62 \\
Median & & \\
Histology & 15 & 20 \\
Adenocarcinoma & 8 & 5 \\
Squamous & & 12 \\
Stage & 13 & 13 \\
IIIb & 10 & $60-90$ \\
IV & & 70 \\
KPS & $60-90$ & \\
Range & 70 & \\
Median &
\end{tabular}

XT, capecitabine combined with docetaxel; T, docetaxel alone; KPS, Karnofsky performance status.

Evaluation criteria of short-term efficacy and adverse events. Response Evaluation Criteria in Solid Tumors (RECIST) version 1.1 for tumor response were used to assess shortterm efficacy in patients (12). The response was evaluated as complete response (CR), partial response (PR), stable disease (SD) and progressive disease (PD). RR was defined as $\mathrm{CR}+\mathrm{PR}$ and the disease control rate (DCR) was defined as $\mathrm{CR}+\mathrm{PR}+\mathrm{SD}$. The response was evaluated every 2 cycles of chemotherapy until death or PD.

Adverse events were evaluated according to the Common Terminology Criteria for Adverse Events v3.0. HFS was graded I to III as defined in a previous capecitabine clinical trial (13).

Subsequent treatment. Subsequent treatment or best supportive treatment following PD was decided by the treating physician.

Follow-up. After treatment, patients were followed up every month for the first half year, every 3 months for the following year, and every 6 months thereafter. Each follow-up included history, physical examination, complete blood count, blood biochemical examination and chest CT.

Death from any cause was calculated from the date of treatment until death or the last follow-up evaluation.

Statistical analysis. The trial was designed as a randomized controlled phase II study. The primary endpoint was TTP and the secondary endpoints were OS, RR and DCR. Analyses were performed using the SPSS 13.0 software package. Constituent ratios were assessed using the $\chi^{2}$ test or Fisher's exact probability test. The means between the two groups were compared using the t-test or rank-sum test. TTP and OS were estimated using the Kaplan-Meier method. Statistical significance was assessed using the log-rank test. $\mathrm{P} \leq 0.05$ was considered to indicate a statistically significant result.
Table II. Hematological toxicities.

\begin{tabular}{lcccc}
\hline & $\begin{array}{c}\text { XT group } \\
\text { cases (\%) }\end{array}$ & $\begin{array}{c}\text { T group } \\
\text { cases }(\%)\end{array}$ & $\chi^{2}$ & P-value \\
\hline Neutropenia & & & & \\
0 & $15(65.2)$ & $16(64.0)$ & & \\
I & $2(8.7)$ & $3(12.0)$ & & \\
II & $4(17.4)$ & $5(20.0)$ & & \\
III & $2(8.7)$ & $1(4.0)$ & 0.008 & 0.930 \\
Anemia & & & & \\
0 & $21(91.4)$ & $22(88.0)$ & & \\
I & $1(4.3)$ & $3(12.0)$ & & \\
II & $1(4.3)$ & $0(0)$ & 0.141 & 0.707 \\
Thrombocytopenia & & & & \\
0 & $21(91.4)$ & $22(88.0)$ & & \\
I & $1(4.3)$ & $1(4.0)$ & & \\
II & $1(4.3)$ & $2(8.0)$ & 0.141 & 0.707 \\
\hline XT, capecitabine combined with docetaxel; T, docetaxel alone. & \\
\hline
\end{tabular}

\section{Results}

Patient characteristics. Between July 2009 and June 2011, 48 sequential previously treated patients with pathologically confirmed NSCLC who failed first-line chemotherapy were enrolled. The patients were randomized into the XT group ( 23 cases) and the T group ( 25 cases). Table I shows the patient characteristics for this study. The 48 patients comprised 29 men and 19 women aged between 48 and 71 years with a median age of 64 years. Thirteen patients had squamous cell carcinoma and 35 had adenocarcinoma. Twenty-five patients had stage IIIb disease and 23 had stage IV disease.

Patients were followed up until death from any cause or the last follow-up evaluation. Until September 30, 2011, no patients were lost to follow up. The median follow up for all 48 patients was 10 months (range, 2-28).

Treatment completion. All 48 patients completed at least 1 cycle of chemotherapy. In the XT group, a total of 78 cycles of chemotherapy were completed (mean of 3.39), and the median number of cycles was 3 (range, 1-6). Prior to treatment, the median number of regimens of applied chemotherapy was 1 (range, 1-3), and the median number of completed chemotherapy cycles was 3 (range, 1-7). In the T group, a total of 88 cycles were completed, with a mean cycle number of 3.52 , and a median cycle number of 3 (range, 1-6). The median number of previous chemotherapy regimens prior to treatment was 1 (range, 1-3), and the median number of chemotherapy cycles completed was 3 (range, 1-6).

Survival data. The median TTP in the XT group was much longer than that in the T group (7 vs. 3 months). The difference was significant for the XT group compared with the T group, as shown in Fig. 1 (log-rank test, $\chi^{2}=4.763, p=0.029$ ). Compared with docetaxel alone, capecitabine combined with docetaxel significantly improved TTP and thus this trial achieved the 
Table III. Non-hematological toxicities.

\begin{tabular}{|c|c|c|c|c|}
\hline & $\begin{array}{l}\text { XT group } \\
\text { cases }(\%)\end{array}$ & $\begin{array}{l}\mathrm{T} \text { group } \\
\text { cases }(\%)\end{array}$ & $\chi^{2}$ & P-value \\
\hline \multicolumn{5}{|c|}{ Hand-foot syndrome } \\
\hline 0 & $13(56.5)$ & $25(100.0)$ & & \\
\hline I & $8(34.8)$ & $0(0)$ & & \\
\hline II & $2(8.7)$ & $0(0)$ & 17.635 & $<0.001$ \\
\hline \multicolumn{5}{|c|}{ Fatigue } \\
\hline 0 & $15(65.2)$ & $21(84.0)$ & & \\
\hline I & $4(17.4)$ & $3(12.0)$ & & \\
\hline II & $2(8.7)$ & $0(0)$ & & \\
\hline III & $2(8.7)$ & $1(4.0)$ & 0.741 & 0.389 \\
\hline \multicolumn{5}{|c|}{ Neuropathy } \\
\hline 0 & $10(43.5)$ & $12(48.0)$ & & \\
\hline I & $8(34.8)$ & $7(28.0)$ & & \\
\hline II & $5(21.7)$ & $6(24.0)$ & 0.099 & 0.753 \\
\hline \multicolumn{5}{|c|}{ Nausea } \\
\hline 0 & $16(69.6)$ & $21(84.0)$ & & \\
\hline I & $4(17.4)$ & $2(8.0)$ & & \\
\hline II & $3(13.0)$ & $2(8.0)$ & 1.413 & 0.235 \\
\hline \multicolumn{5}{|c|}{ Alopecia } \\
\hline 0 & $13(56.4)$ & $11(44.0)$ & & \\
\hline I & $10(43.5)$ & $14(56.0)$ & 0.751 & 0.386 \\
\hline \multicolumn{5}{|c|}{ Peripheral edema } \\
\hline 0 & $15(65.2)$ & $16(64.0)$ & & \\
\hline I & $6(26.1)$ & $8(32.0)$ & & \\
\hline II & $2(8.7)$ & $1(4.0)$ & 0.008 & 0.930 \\
\hline \multicolumn{5}{|c|}{ Vomiting } \\
\hline I & $1(4.3)$ & $0(0)$ & a & 0.479 \\
\hline \multicolumn{5}{|c|}{ Joint pain } \\
\hline I & $2(8.7)$ & $4(16.0)$ & 0.596 & 0.440 \\
\hline
\end{tabular}

${ }^{\mathrm{a} F i s h e r ' s ~ e x a c t ~ p r o b a b i l i t y ~ t e s t . ~}$

primary endpoint. The median survival time (MST) and 1-year OS rate in the XT group and T group were 12 months, $47.6 \%$ and 12 months, $39.6 \%$, respectively. No difference in overall survival was observed between the XT and T group, as shown in Fig. 2 (log-rank test, $\left.\chi^{2}=0.446, \mathrm{p}=0.504\right)$.

Short-term response. Cases of $\mathrm{CR}, \mathrm{PR}, \mathrm{SD}$ and $\mathrm{PD}$ for the patients in the XT group and T group were $0,3,15,5$ and 0,3 , 16,6 , respectively. The RR and DCR in the XT and T groups were $13.0 \%$ (3/23), $78.3 \%$ (18/23) and $12.0 \%$ (3/25), $76.0 \%$ (19/25), respectively. No difference in short-term response was noted between the XT and T groups ( $\mathrm{p}>0.05$ ).

Hematological toxicity. The hematological toxicities observed are shown in Table II; most were mild to moderate and manageable clinically. Only 6.25\% (3/48) of the patients had grade III neutropenia without fever. No grade III or higher anemia and thrombocytopenia occurred. No difference in the rates for neutropenia, anemia and thrombocytopenia was noted between the XT and T groups ( $>0.05)$.
Non-hematological toxicity. The non-hematological toxicities are shown in Table III. Severe non-hematological toxicities were rare; the only grade III toxicity was fatigue, which occurred in 2 patients in the XT group. Although the HFS rate was significantly higher in the XT group compared to the T group (43.5 vs. $0 \%$ ), no grade III HFS was observed in the XT group and only 1 patient experienced grade II HFS in the XT group. The rates of fatigue and nausea were higher in the XT group than in the T group (34.8 vs. $16 \%$ and 30.4 vs. $16 \%$, respectively). The difference was not significant. Incidences of neuropathy, alopecia and peripheral edema were almost the same in the two groups. No hyperbilirubinemia was observed in any of the 48 patients.

\section{Discussion}

Subsequent chemotherapy following first-line treatment of NSCLC has always been difficult, and treatment efficacy has been poor. Although the second-line treatment recommended by NCCN guidelines in the USA utilized a single agent, treat- 


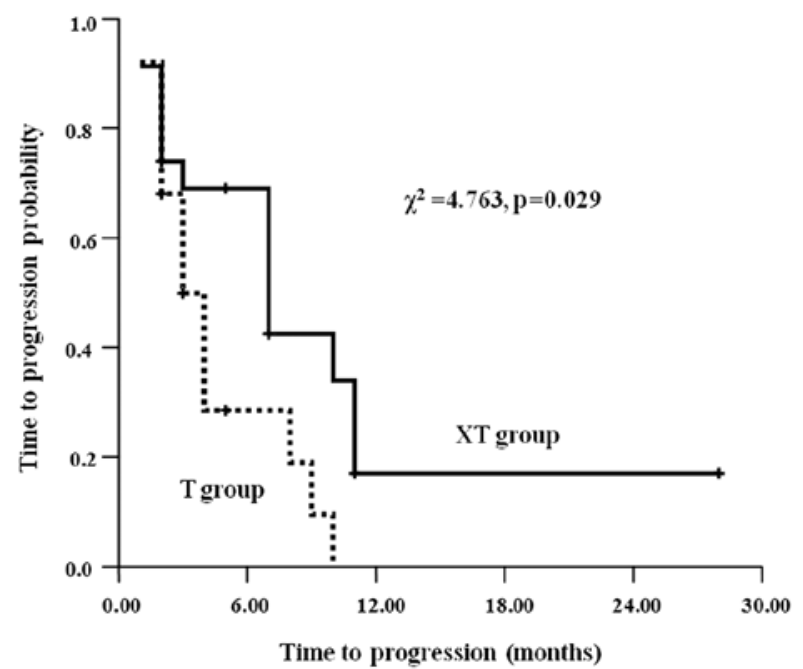

Figure 1. Median time to progression (TTP) in the XT group and the T group was 7 and 3 months, respectively. The difference was significant (log-rank test, $\left.\chi^{2}=4.763, \mathrm{p}=0.029\right)$. XT, capecitabine combined with docetaxel; $\mathrm{T}$, docetaxel alone.

ment efficacy using only one agent has been limited. Therefore, exploring the effect of a combination treatment regimen of two agents following first-line therapy has important clinical significance.

Docetaxel was widely studied in the second-line treatment of NSCLC, and it was accepted as the standard treatment $(4,14)$. Therefore, most combination treatment regimens of two agents were based on docetaxel with platinum or another chemotherapy agent. Although there have been several studies on the two-drug treatment of docetaxel, all of these were phase I and II trials, and yielded inconsistent results. Certain studies reported that a two-agent regimen based on docetaxel was better than docetaxel alone and significantly improved TTP or progression-free survival (PFS) (15-17), while different results were yielded in another study. Takeda et al reported that the RR, PFS and OS observed with docetaxel alone were similar to those observed using the combination treatment (18). Therefore, there was no consensus for two-agent regimens of docetaxel as a second-line treatment of NSCLC.

Capecitabine was the precursor of fluorouracil (FU). Following absorption, the drug can be converted into FU by a three-step enzyme cascade reaction to play an antitumor role. In addition, TP was found to be a type of rate-limiting enzyme. As tumor tissues highly express TP, capecitabine at a high concentration accumulates in tumor tissues and has a certain targeted tumor-killing effect (19). It has been proven that capecitabine has strong treatment efficacy in colorectal, gastric, breast and ovarian cancer. $(4,6)$. Animal experiments showed that capecitabine had a certain antitumor effect on NSCLC, and that docetaxel was capable of upregulating TP activity (6). In addition, capecitabine and docetaxel had a synergistic effect. Theoretically, a combination application of the two agents may achieve a higher treatment efficacy than docetaxel alone.

Certain clinical trials preliminarily confirmed the treatment efficacy of capecitabine combined with docetaxel in previously treated NSCLC patients. Kindwall-Keller et al (7) reported a phase II trial of capecitabine plus docetaxel: RR,

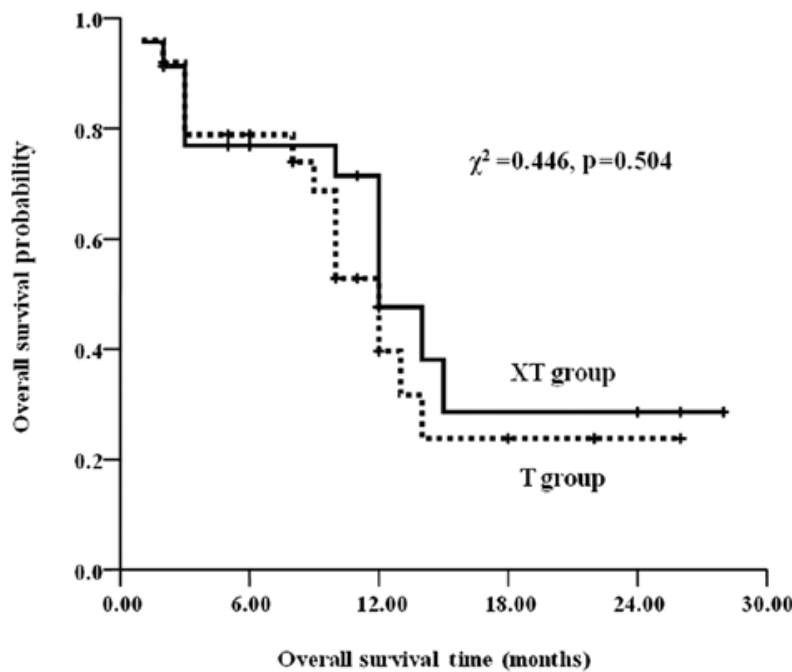

Figure 2. Median survival time (MST) and 1-year overall survival (OS) rate in the XT group and T group were 12 months, $47.6 \%$ and 12 months, 39.6\%, respectively. No difference in overall survival was observed between the two groups (log-rank test, $\left.\chi^{2}=0.446, \mathrm{p}=0.504\right)$. XT, capecitabine combined with docetaxel; T, docetaxel alone.

6-month PFS rate, MST and 1-year OS rate were 26\%, 25\%, 9.1 months and $37 \%$, respectively. In addition, the combination regimen was well-tolerated. Therefore, the authors suggested the application of a randomized controlled trial to evaluate the treatment regimen of capecitabine combined with docetaxel. Similar treatment efficacy is found in studies on the Oriental population. Lee et al (8) reported a phase II trial in Korea. The RR was $15.4 \%$, and the MST and 1-year OS rate were 8.7 months and $34.4 \%$, respectively. Although the preliminary efficacy of capecitabine plus docetaxel was reported, there was no randomized controlled study on its long-term survival result. The final survival results of the two-agent regimen remain unclear.

Liu et al (9) reported the small-sample single group study of docetaxel plus FU as a second-line treatment for advanced NSCLC. Capecitabine plus docetaxel was used to treat 8 patients. Of the 8 patients, 2 patients achieved PR and 3 achieved SD. The DCR was $62.5 \%$. Survival data were not available. To the best of our knowledge, this is the first randomized controlled study to comparatively evaluate the treatment efficacy of capecitabine combined with docetaxel. Our results showed that this trial achieved the primary endpoint, i.e., that capecitabine combined with docetaxel significantly improved TTP compared with docetaxel alone and obtained good treatment efficacy, with an RR of 13\%, median TTP of 7 months and 1-year OS rate of $47.6 \%$, which had good comparability to similar studies. In those similar studies, the RR range, median TTP and 1-year OS rate were 6.7-22.3\%, 4-5.6 months and $37.0-46 \%(15,18,20,21)$, respectively. Since the sample size in our study was small and the follow-up time was relatively short, long-term survival still requires further observation.

Since palliation is the most important aim for previously treated patients with NSLCC, it is reasonable to select a dose with lower toxicity while conserving its anticancer activity. Our findings have shown that capecitabine combined with docetaxel had only mild to moderate side effects and good tolerance. Compared with docetaxel alone, hematological 
toxicity, nausea, vomiting, fatigue and other side effects did not significantly increase. Combination administration only increased morbidity of HFS (43.5\% and 0 ), but there were no severe cases of HFS. No grade III HFS occurred, and only 1 case of grade II HFS was observed (4.3\%). No dose reduction was caused by HFS, suggesting that this combination regimen was well-tolerated and practical for clinical implementation. Compared with other similar studies, the incidence of HFS in our study was fairly low, and severe fatigue, neutropenia and anemia were milder $(7,8)$.

In conclusion, capecitabine combined with docetaxel significantly improved TTP compared with docetaxel alone for previously treated NSCLC patients, and this regimen also achieved a good 1-year survival rate and DCR. The side effects of this regimen are mild to moderate. A phase III trial is required to further evaluate the long-term survival.

\section{Acknowledgements}

This study was supported by a grant from the Science and Technology Department of Hebei Province, China (No. 072761711).

\section{References}

1. Ferlay J, Shin HR, Bray F, Forman D, Mathers C and Parkin DM: Estimates of worldwide burden of cancer in 2008: GLOBOCAN 2008. Int J Cancer 127: 2893-2917, 2010.

2. Parkin DM, Bray F, Ferlay J and Pisani P: Global cancer statistics, 2002. CA Cancer J Clin 55: 74-108, 2005.

3. http://www.nccn.org/professionals/physician_gls/f_guidelines.asp

4. Comer AM and Goa KL: Docetaxel: a review of its use in non-small cell lung cancer. Drugs Aging 17: 53-80, 2000.

5. O'Shaughnessy J, Miles D, Vukelja S, Moiseyenko V, Ayoub JP, Cervantes G, Fumoleau P, Jones S, Lui WY, Mauriac L, et al: Superior survival with capecitabine plus docetaxel combination therapy in anthracycline-pretreated patients with advanced breast cancer: phase III trial results. J Clin Oncol 20: 2812-2823, 2002.

6. Sawada N, Ishikawa T, Fukase Y, Nishida M, Yoshikubo T and Ishitsuka $\mathrm{H}$ : Induction of thymidine phosphorylase activity and enhancement of capecitabine efficacy by taxol/taxotere in human cancer xenografts. Clin Cancer Res 4: 1013-1019, 1998.

7. Kindwall-Keller T, Otterson GA, Young D, Neki A, Criswell T, Nuovo G, Soong R, Diasio R and Villalona-Calero MA: Phase II evaluation of docetaxel-modulated capecitabine in previously treated patients with non-small cell lung cancer. Clin Cancer Res 11: 1870-1876, 2005.

8. Lee JJ, Han JY, Lee DH, Kim HY, Chun JH, Lee HG, Yoon SM, Lee SY and Lee JS: A phase II trial of docetaxel plus capecitabine in patients with previously treated non-small cell lung cancer. Jpn J Clin Oncol 36: 761-767, 2006.
9. Liu ZH, Liao XL, Zhou WX, Li YQ, Lu YK, Tan XH, Xie WM and $\mathrm{Hu} \mathrm{XH}$ : Phase II trial of docetaxel combined with fluoropyrimidines as the second line therapy for patients with non-small cell lung cancer. Acta Medicine of GuangXi Medical University 25: 706-708, 2008 (In Chinese).

10. Gervais R, Ducolone A, Breton JL, Braun D, Lebeau B, Vaylet F, Debieuvre D, Pujol JL, Tredaniel J, Clouet P and Quoix E: Phase II randomised trial comparing docetaxel given every 3 weeks with weekly schedule as second-line therapy in patients with advanced non-small-cell lung cancer (NSCLC). Ann Oncol 16: 90-96, 2005.

11. Lai CL, Tsai CM, Chiu CH, Wang GS, Su WJ, Chen YM and Perng RP: Phase II randomized trial of tri-weekly versus days 1 and 8 weekly docetaxel as a second-line treatment of advanced non-small cell lung cancer. Jpn J Clin Oncol 35: 700-706, 2005.

12. Eisenhauer EA, Therasse P, Bogaerts J, Schwartz LH, Sargent D, Ford R, Dancey J, Arbuck S, Gwyther S, Mooney M, et al: New response evaluation criteria in solid tumours: revised RECIST guideline (version 1.1). Eur J Cancer 45: 228-247, 2009.

13. Blum JL, Jones SE, Buzdar AU, LoRusso PM, Kuter I, Vogel C, Osterwalder B, Burger HU, Brown CS and Griffin T: Multicenter phase II study of capecitabine in paclitaxel-refractory metastatic breast cancer. J Clin Oncol 17: 485-493, 1999.

14. Davies AM, Lara PN Jr, Mack PC and Gandara DR: Docetaxel in non-small cell lung cancer: a review. Expert Opin Pharmacother 4: $553-565,2003$

15. Pectasides D, Pectasides M, Farmakis D, Kostopoulou V, Nikolaou M, Gaglia A, Koumpou M, Mylonakis N, Xiros N, Economopoulos T and Raptis SA: Comparison of docetaxel and docetaxel-irinotecan combination as second-line chemotherapy in advanced non-small-cell lung cancer: a randomized phase II trial. Ann Oncol 16: 294-299, 2005.

16. Zhang YF, Yu YF and Lu S: Comparison of single-agent docetaxel versus docetaxel plus platinum combination agent in second-line treatment for advanced non-small cell lung cancer. Natl Med J China 89: 1544-1548, 2009 (In Chinese).

17. Gu LP, Shen SP and Chen ZW: Comparison of the efficacy of nedaplatin combined with docetaxel and docetaxel alone as a second line treatment for advanced non-small cell lung cancer. Chin J Oncol 32: 939-942, 2010 (In Chinese).

18. Takeda K, Negoro S, Tamura T, Nishiwaki Y, Kudoh S, Yokota S, Matsui K, Semba H, Nakagawa K, Takada Y, et al: Phase III trial of docetaxel plus gemcitabine versus docetaxel in secondline treatment for non-small-cell lung cancer: results of a Japan Clinical Oncology Group trial (JCOG0104). Ann Oncol 20: 835-841, 2009.

19. Miwa M, Ura M, Nishida M, Sawada N, Ishikawa T, Mori K, Shimma N, Umeda I and Ishitsuka H: Design of a novel oralfluoropyrimidine carbamate, capecitabine, which generates 5 -fluorouracil selectively in tumors by enzymes concentrated in human liver and cancer tissue. Eur J Cancer 34: 1274-1281, 1998.

20. Liu SL, Gu SX, Shen SJ, Tao YH and Jing C: Effect of weekly administration of docetaxel combined with oxaliplatin as secondline therapy on non-small cell lung cancer. Chin Clin Oncol 15: 749-751, 2010 (In Chinese).

21. Liu Y and Cheng Y: The clinical study of weekly docetaxel and cisplatin as a second-line therapy for recurrent or progressive non-small cell lung cancer. Oncol Progress 6: 528-531, 2008 (In Chinese). 